\title{
Es kann losgehen!
}

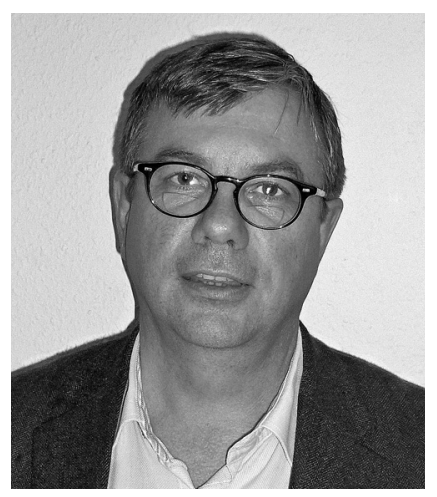

VON ANDREAS BARTELS

Andreas Bartels ist Referent für Europa bei der Arbeiterwohlfahrt Bundesverband e. V. in Berlin. www.awo.org

\author{
Die neue Förderperiode des Europäischen \\ Strukturfonds läuft von 2014 bis 2020. Trotz großer \\ Einschnitte können auch soziale Organisationen und \\ Unternehmen der Sozialwirtschaft in Deutschland \\ wieder von dem Geld aus Brüssel profitieren.
}

Viele in Europa haben lange darauf gewartet, dass die Verhandlungen zwischen Parlament, Rat und Kommission zu den Strukturfonds der Europäischen Union und zum Mittelfristigen Finanzrahmen endlich zum erfolgreichen Abschluss kommen. Nach zahlreichen »Trilogsitzungen« in Brüssel sind der Mittelfristige Finanzrahmen und die Verordnungen zu den EU-Strukturfonds am 19. November 2013 im Europäischen Parlament und am 2. Dezember 2013 im Rat verabschiedet worden.

Nun kann an der Umsetzung der Strukturfonds in den Mitgliedsstaaten gearbeitet werden, die zunächst vorsieht, dass mit der Europäischen Kommission eine Partnerschaftsvereinbarung und darauf aufbauend die Operationellen Programme eingereicht, geprüft und genehmigt werden, bevor die darin eingebetteten Programme des Europäischen Sozialfonds (ESF) für die neue Förderperiode 2014 bis 2020 beginnen können.

\section{Wie alles begann}

Ein weiter Weg liegt zwischen der Verabschiedung der Strukturfonds und dem Mittelfristigen Finanzrahmen! Im Oktober 2010 legte die Europäische Kommission die »EU 2020-Strategie « vor, welche die gescheiterte LissabonStrategie, die die Europäische Union bis 2010 zum wettbewerbsfähigsten und dynamischsten wissensgestützten Wirtschaftsraum der Welt machen sollte, ablöst und für das nächste Jahrzehnt die Wachstumsstrategie der Europäischen Union darstellt.
Durch die - auch vom Europäischen Parlament und vom Europäischen Rat unterstützte - Strategie sollen bis 2020 u. a. folgende sozialpolitischen Ziele erreicht werden: Beschäftigung fördern, Bildungsniveau verbessern, soziale Eingliederung vor allem durch Armutsbekämpfung fördern. Zu den EU-weiten Zielvorgaben hat ein jedes Mitgliedsland im Rahmen seines Nationalen Reformprogrammes seine Beiträge dazu einzubringen.

Deutschland hat sich dementsprechend zu nationalen Indikatoren bei den sozialpolitischen Zielen verpflichtet, die zum Teil wenig ambitioniert sind (z. B. bei der Senkung der Anzahl der Personen in Armut) und zum Teil bereits erreicht wurden, bevor die neue Förderperiode der Strukturfonds überhaupt beginnt. Die neue Förderphase soll sich stärker an den strategischen Zielen der »EU 2020-Strategie " ausrichten und somit eine stärkere Verpflichtung zu vorher festgelegten Ergebnissen eingehen.

Ein Jahr später im Oktober 2011 veröffentlichte die Europäische Kommission das Verordnungspaket für die Strukturfonds. Diese wurden zur weiteren Erörterung und Bescheidung an das Europäische Parlament und den Rat überwiesen. Die wesentlichen Ergebnisse aus den Trilogverhandlungen:

- Alle Strukturfonds berücksichtigen die horizontalen Prinzipien: Gleichstellung der Geschlechter, Nichtdiskriminierung, Nachhaltigkeit.

- Das Partnerschaftsprinzip konnte nachhaltig gestärkt werden: Kommunen, Wirtschafts- und Sozialpartner 


\section{Europa 2020 und das deutsche NRP}

\begin{tabular}{|c|c|c|}
\hline Zielbereiche & Ziele EU 2020 & Ziele deutsches NRP \\
\hline $\begin{array}{l}\text { Beschäftigung } \\
\text { (Beschäftigungsquote: } \\
\text { Beschäftigte im Verhältnis zu } \\
\text { Erwerbsfähigen im Alter von } \\
\text { 20-64) }\end{array}$ & $\begin{array}{l}\text { Erhöhung der Quote auf } \\
\qquad 75 \% \\
\text { (plus Ziele für Ältere und } \\
\text { Frauen) }\end{array}$ & $\begin{array}{c}77 \% \text { (3. Quartal 2012: 77,1\%) } \\
\text { Ältere (55-64): 60\% } \\
\text { (3. Quartal 2012: 62,1\%) } \\
\text { Frauen: } 73 \% \\
\text { (3. Quartal 2012: } 71,7 \%)\end{array}$ \\
\hline $\begin{array}{c}\text { Bildung } \\
\text { (Schulabbrecher ohne Bildung } \\
\text { / Ausbildung im Alter 18-24) } \\
\text { (Erfolgreiche Teilnahme an } \\
\text { tertiärer Bildung im Alter } 30 \text { - } \\
\text { 34) }\end{array}$ & $\begin{array}{c}\text { Senkung der } \\
\text { "Abbrecherquote" auf } \\
\text { unter } 10 \% \\
\text { Erhöhung der } \\
\text { Teilnahmequote auf } 40 \%\end{array}$ & $\begin{array}{c}\text { Senkung der } \\
\text { „Abbrecherquote" auf unter } \\
\text { 10\% (2011: } 11,5 \%) \\
\text { Erhöhung der Teilnahmequote } \\
\text { auf } 42 \%(2011: 42,2 \%)\end{array}$ \\
\hline $\begin{array}{l}\text { Armut und soziale } \\
\text { Ausgrenzung } \\
\text { Drei Dimensionen: } \\
\text { (1) Relative Einkommens- } \\
\text { armut; (2) Materielle } \\
\text { Deprivation; (3) Menschen in } \\
\text { Haushalten mit geringer } \\
\text { Erwerbsintensität }\end{array}$ & $\begin{array}{c}\text { Senkung der } \\
\text { Armutsbevölkerung um } \\
20 \% \text { (von } 116 \text { Mio.) }\end{array}$ & $\begin{array}{l}\text { Senkung der Bevölkerung in } \\
\text { Haushalten von Langzeit- } \\
\text { arbeitslosen (2008: } 1,63 \text { Mio. } \\
\text { Langzeitarbeitslose; Verringe- } \\
\text { rung LZA um 20\%: } 330.000 \\
\text { 2008-2012: Verringerung um } \\
430.000 \text { Personen }\end{array}$ \\
\hline
\end{tabular}

und die Vertreter der Zivilgesellschaft sind an der Planung, Umsetzung und Evaluierung der Strukturfonds zu beteiligen und nehmen gleichberechtigt an den Begleitausschüssen teil. Erstmals gibt es in der Verordnung Mindeststandards für die Beteiligung der Partner und ein verbindliches Handbuch für gute demokratische Praxis.

- Elf thematische Ziele sind für alle Strukturfonds vorgegeben. Für den Europäischen Sozialfonds hat man sich auf die folgenden drei Ziele verständigt: (8) Förderung von nachhaltiger und qualitativer Beschäftigung und Unterstützung der Mobilität der Arbeitskräfte (9) Förderung der sozialen Eingliederung und Bekämpfung der Armut und Diskriminierung (10) Investitionen in Bildung, Training einschließlich Ausbildung, Kompetenzen und lebenslanges Lernen.

- Europäisches Parlament und Europäischer Rat haben sich auf den Mittelfristigen Finanzrahmen für die Jahre 2014 bis 2020 verständigt. Demnach stehen für die Kohäsionspolitik insgesamt 325,25 Milliarden Euro zur Verfügung. Davon können 23,1 Prozent (also 75,13 Milliarden Euro) europaweit für den Europäischen Sozialfonds eingesetzt werden. 20 Prozent davon sind verpflichtend für das thematische Ziel Armutsbekämpfung vorzusehen.

- Für Deutschland ergibt sich daraus folgendes Finanztableau: Aufgrund der positiven Wirtschaftsentwicklung und des insgesamt gekürzten Mittelfristigen Finanzrahmen stehen ca. 35 Prozent weniger Mittel aus den Strukturfonds zur Verfügung. Von den insgesamt 17,15 Milliarden Euro sind 5,66 Milliarden Euro für den Europäischen Sozialfonds vorgesehen. Für das Operationelle Programm des Bundes sind dies 2,46 Milliarden Euro.

\section{Die neue Förderperiode in Deutschland}

Aufgrund der deutlich weniger zur Verfügung stehenden Mittel für die Strukturfonds in Deutschland und der strategischen
Ausrichtung auf die »EU Strategie 2020 « sind umfassende Konsultationsprozesse (unter Einbezug der Partner) durchgeführt worden, mit dem Ziel, die zur Verfügung stehenden Mittel zu konzentrieren. Parallel dazu haben Kohärenzgespräche zwischen dem Bund und den Ländern stattgefunden, um künftig Doppelfinanzierungen von Programmen zu vermeiden. Beide Prozesse konnten erfolgreich abgeschlossen worden, so dass nun die Vorbereitungen der Partnerschaftsvereinbarungen und der Operationellen Programme in die finale Phase gehen.

Im Ergebnis gibt es künftig anstatt bisher 61 ESF-Bundesprogramme, aufgeteilt auf vier Ministerien, nur noch 27 Programme, aufgeteilt auf fünf Ministerien. Das Bundesumweltministerium ist als neuer Akteur dabei. Die Planungen für die neue Programmgeneration sind bereits weit vorangeschritten. Das Bundesministerium für Arbeit und Soziales plant neun ESF-Programme, davon zwei Partnerschaftsprogramme, und das Bundesministerium für Familie, Senioren, Frauen und Jugend hat sich auf sechs Programmschwerpunkte konzentriert. Alle Programme adressieren die genannten drei thematischen Ziele des Europäischen Sozialfonds.

\section{Das neue Partnerschaftsprogramm in der Sozialwirtschaft}

In guter Kooperation mit dem Bundesministerium für Arbeit und Soziales hat sich die Bundesarbeitsgemeinschaft der

\section{ESF 2014-2020 im BMAS}

\begin{tabular}{|c|c|}
\hline $\begin{array}{c}\text { Prioritätsachse / } \\
\text { Investitionspriorität }\end{array}$ & $\begin{array}{c}\text { Spezifische Ziele / } \\
\text { Angedachte Programme }\end{array}$ \\
\hline $\begin{array}{l}\text { Prioritätsachse A: Förderung von Beschäftigung } \\
\text { und Unterstützung der Mobilität der Arbeitskräfte }\end{array}$ & $\begin{array}{l}\text { - Verankerung systematischer Weiterbildung in } \\
\text { Unternehmen, Organisationen und Branchen } \\
\text { Angedachte Programme: Sozialpartnerrichtlinie, } \\
\text { Partnerrichtlinie Wohlfahtsverbände } \\
\text { - Aufbau und Sicherung von Beschäftigung und } \\
\text { der Anpassungsfähigkeiten in KMU's an den } \\
\text { Wandel } \\
\text { Angedachtes Programm: Qualikug Transfer } \\
\text { - Aktivierung von bislang ungenutztem } \\
\text { Fachkräfteangebot } \\
\text { Angedachte Programme: Vollzeitnah statt } \\
\text { unterbeschäftigt, Qualifizierungsprogramm für } \\
\text { Migrantinnen und Migranten im Kontext des } \\
\text { Anerkennungsgesetzes }\end{array}$ \\
\hline
\end{tabular}


ESF 2014-2020 im BMAS

\begin{tabular}{|c|c|}
\hline $\begin{array}{c}\text { Prioritätsachse I } \\
\text { Investitionspriorität }\end{array}$ & \multicolumn{1}{c|}{$\begin{array}{c}\text { Spezifische Ziele } \text { I } \\
\text { Angedachte Programme }\end{array}$} \\
\hline & $-\begin{array}{l}\text { Integration von Langzeitarbeitslosen in den } \\
\text { Arbeitsmarkt } \\
\text { Angedachtes Programm: Bundesprogramm für } \\
\text { Pangzeitarbeitslose }\end{array}$ \\
Eingliedsachse B: Förderung der sozialen & $-\begin{array}{l}\text { Vermittlung von Migranten/innen in Arbeit, } \\
\text { Ausbildung oder Qualifizierung } \\
\text { Angedachte Programme: Integrationsrichtlinie } \\
\text { Bund, Sprachförderung für Menschen mit } \\
\text { Migrationshintergrund }\end{array}$ \\
\hline & $-\begin{array}{l}\text { Eingliederung junger Menschen in eine } \\
\text { Berufsausbildung }\end{array}$ \\
& Angedachtes Programm: \\
& Berufseinstiegsbegleitung \\
\hline Prioritätsachse C: Investition in Bildung, & \\
\hline Kompetenzen und lebenslanges Lernen & \\
\hline
\end{tabular}

Freien Wohlfahrtspflege mit zwei Programmvorschlägen in die Diskussion um die Ausrichtung des Europäischer Sozialfonds 2014 bis 2020 eingebracht: einen Vorschlag für die Beschäftigten in der Sozialwirtschaft mit dem Schwerpunkt Personal- und Organisationsentwicklung zur Gestaltung des demografischen Wandels und ein weiterer zur Armutsbekämpfung, der sich auf gesellschaftliche und kulturelle Teilhabe und die Stärkung der eigenen Beschäftigungsfähigkeit für stark benachteiligte Personen (z. B. Wohnungslose), konzentriert.

Die Verhandlungen haben dazu geführt, dass eine Partnerrichtlinie für die Wohlfahrtsverbände verfolgt wird, die eine konsequente Fortführung des Programms »rückenwind - Für die Beschäftigten in der Sozialwirtschaft " darstellt. Im Rahmen dieser Richtlinie sollen integrierte Vorhaben zur Personalund Organisationsentwicklung in der Sozialwirtschaft in folgenden Bereichen gefördert werden:

A. Personalentwicklung zur Verbesserung der Anpassungs- und Beschäftigungsfähigkeit:

a altersgerechte Personalentwicklung

- berufsbegleitende Qualifizierung, Beratung und Coaching

- Maßnahmen zum Erhalt der Beschäftigungsfähigkeit insbesondere älterer Arbeitnehmer/innen

- Einstiegs- und Anpassungsqualifizierungen
- Personalgewinnung: Kampagnen zur Nachwuchsgewinnung, Begleitung und Perspektiventwicklung von an der Sozialwirtschaft interessierten Personen

- Personalentwicklung im Sozialraum

B. Organisationsentwicklung zur Verbesserung der Demografiefestigkeit sozialwirtschaftlicher Organisationen:

- gesundheitsfördernde Arbeitsbedingungen

- Vereinbarkeit von Familie und Beruf

- Erhöhung des Anteils von Frauen in Führungspositionen
- Begleitung von Quer- sowie Wiedereinsteigerinnen und Wiedereinsteigern

- Führung und Unternehmenskultur

- Entwicklung von lebensphasenorientierten Arbeitszeitmodellen

- Diversity Management und Förderung der Inklusionsfähigkeit

- Förderung der Innovationsfähigkeit (auch im Hinblick auf regionale Abstimmungsprozesse)

Das neue Programm für die Sozialwirtschaft hat sich einigen Neuerungen hinsichtlich der Förderbedingungen und der Fördertechnik verschrieben:

- Projektvorschläge müssen einen integrativen Ansatz zur Personal- und Organisationsentwicklung verfolgen.

- Antragsteller orientieren sich an den für die Branche üblichen Tarifen oder weisen eine tarifähnliche Regelung nach und halten sich an die vereinbarten Mindestlöhne.

- Mindestens fünf Prozent der Gesamtkosten des Projektes müssen in Form von Eigenmitteln (Barmittel) oder Drittmitteln erbracht werden.

- Die Projekte müssen Informationen zu den in der Verordnung zum Europäischen Sozialfonds genannten Output-Indikatoren liefern.

- Darüber hinaus werden für das Programm Ergebnisindikatoren festgelegt, die die Projektträger bedienen müssen: z. B. die Erhöhung der Anzahl von älteren Beschäftigten (ab 54 Jahre) in den beteiligten Unternehmen.

\section{ESF 2014-2020 im BMFSFJ}

\begin{tabular}{|c|c|}
\hline $\begin{array}{c}\text { Prioritätsachse } \\
\text { Investitionspriorität }\end{array}$ & Spezifische Ziele / Programme \\
\hline $\begin{array}{l}\text { Prioritätsachse A: Förderung von } \\
\text { Beschäftigung und Unterstützung der } \\
\text { Mobilität der Arbeitskräfte }\end{array}$ & $\begin{array}{l}\text { - Unterstützung der Vereinbarkeit von Berufs- und } \\
\text { Privatleben } \\
\text { Angedachte Programme: Vereinbarkeit von Familie } \\
\text { und Beruf gestalten: Unternehmensnetzwerk/Lokale } \\
\text { Bündnisse für Familie, Quereinstieg: Mehr Männer } \\
\text { in Kitas, Elternchance II: Familien früh für Bildung } \\
\text { gewinnen } \\
\text { - Förderung einer familienfreundlichen Personalpolitik } \\
\text { und Unterstützung beim beruflichen (Wieder)- } \\
\text { Einstieg } \\
\text { Angedachte Programme: Perspektive } \\
\text { Wiedereinstieg, Ressourcen stärken - Zukunft } \\
\text { sichern: Erwerbsperspektiven für Mütter mit } \\
\text { Migrationshintergrund }\end{array}$ \\
\hline $\begin{array}{l}\text { Prioritätsachse B: Förderung der sozialen } \\
\text { Eingliederung und Bekämpfung der Armut }\end{array}$ & $\begin{array}{l}\text { - Verbesserung des Zugangs zu Beschäftigung, } \\
\text { Ausbildung und Bildung für Benachteiligte, } \\
\text { insbesondere junge Menschen } \\
\text { Angedachtes Programm: Jugend stärken im } \\
\text { Quartier }\end{array}$ \\
\hline
\end{tabular}


- Bei der Finanzierung von Projektvorhaben wird an einen Interventionssatz ohne weiteren Nachweis der Größe des Unternehmens gedacht $(50 \%$ oder $60 \%)$.

- Eingeführt werden Pauschalen für indirekte und direkte Kosten (Bezugsgröße sind die direkten Personalkosten sowie reine Honorarkosten).

- Eingeführt werden Standardeinheitssätze für Teilnehmereinkommen oder Freistellungskosten.

- Die Laufzeit der Projekte soll zwei bis drei Jahre sein; nach 18 Monaten findet eine Überprüfung der angegebenen Meilensteine und Ergebnisse statt, bevor eine Weiterförderung ausgesprochen wird.

\section{Informationen im Internet}

Aktuelle Hinweise über den Stand der Verhandlungen zum Europäischen Strukturfonds sind auf den folgenden Webseiten zu finden:

Europäisch: http://www.elisabeth-schroedter.de/themen/zukunft-der-kohaesionspolitik National: http://www.esf.de/portal/generator/20280/foerderperiode__2014-2020.html

Beschäftigtenprojekte vor: Absenkung der Interventionssätze und Nicht-Anerkennung von Personalkosten der Ausbildungsteilnehmer (Freistellungskosten) und der allgemeinen indirekten Kosten (Verwaltungskosten, Miete, Gemeinkosten).

Eine kontinuierliche Intervention bei der zuständigen Generaldirektion Wettbewerb durch die Bundesarbeitsgemein-

\section{"Ab Herbst 2014 ist mit der Umsetzung der neuen Programme zu rechnen«}

Sowohl diese Richtlinie als auch die der Sozialpartner unterliegen dem Beihilferecht. Die dafür vorgesehene "Allgemeine Gruppenfreistellungsverordnung « wird von der Europäischen Kommission gerade überarbeitet und sieht eklatante Verschlechterungen für die über den Europäischer Sozialfonds geförderten schaft der Freien Wohlfahrtspflege hat zwischenzeitlich immerhin schon dazu geführt, dass die Verschlechterungen zum Teil zurückgenommen wurden: nun können kleine und mittlere Unternehmen Freistellungskosten und indirekte Kosten wieder bei der Finanzierung von Projektvorhaben nutzen. Einrichtungen über 250 Mitarbeiter sind aber weiter draußen vor - und in der Freien Wohlfahrtspflege gibt es viele Einrichtungen, die über 250 Mitarbeiter beschäftigen. Diese sollen aber auch künftig weiterhin die Möglichkeit erhalten, sich an dem Programm beteiligen zu können, um den demografischen Wandel in der eigenen Einrichtung mitzugestalten.

Hierzu sind weitere Bemühungen von Seite der Freien Wohlfahrtspflege nötig, damit das neue Partnerschaftsprogramm ein ebenso erfolgreiches wird wie sein Vorgängerprogramm. Der aktuelle Zeitplan sieht vor, dass bis Juni 2014 das Genehmigungsverfahren für die Partnerschaftsvereinbarung und das Operationelle Programm des Bundes für den Europäischen Sozialfonds durch die Europäische Kommission abgeschlossen sein könnte. Danach, ab September 2014, ist mit der Umsetzung der neuen Programme im Europäischen Sozialfonds zu rechnen.

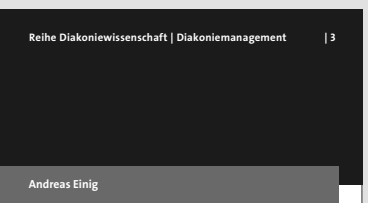

Wie im Himmel so auf Erden

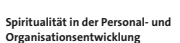

Tomos

\section{Wie im Himmel so auf Erden}

Spiritualität in der Personal- und Organisationsentwicklung

Von Andreas Einig

2014, 358 S., brosch., 39,-€, ISBN 978-3-8487-0978-6

(Reihe Diakoniewissenschaft/Diakoniemanagement, Bd. 3)

Spiritualität ermöglicht neben vordergründigen Lern- und Entwicklungsprozessen den Wandel aus dem Transzendenzbezug heraus. Um dies bei der Entwicklung von Personen, Teams sowie Organisationen zu ermöglichen, zeigt der Verfasser, wie Spiritualität im Managementmodell verortet und in Instrumenten der Personal- und Organisationsentwicklung integriert werden kann. 